Chinese Journal of Astronomy and Astrophysics manuscript no.

(HATEX: zc.tex; printed on March 12, 2021; 20:23)

\title{
The relation between black hole masses and Lorentz factors of the jet components in blazars
}

\author{
Ming Zhou ${ }^{1,2,3 \star}$ and Xinwu Cao ${ }^{1,2 \star}$ \\ 1 Shanghai Astronomical Observatory, CAS, Shanghai 200030, China \\ ' 2 Joint Institute for Galaxy and Cosmology (JOINGC) of SHAO and USMC, 80 \\ Randan Road, Shanghai 200030, China \\ 3 Graduate School of the Chinese Academy of Sciences, BeiJing 100039, China
}

Received month day; accepted month day

\begin{abstract}
We explore the relation between black hole mass $\left(M_{\mathrm{BH}}\right)$ and the motion of the jet components for a sample of blazars. The Very Long Baseline Array (VLBA) 2cm Survey and its continuation: Monitoring of Jets in active galactic nuclei (AGNs) with VLBA Experiments (MOJAVE) have observed 278 radio-loud AGNs, of which 146 blazars have reliable measurements on their apparent velocities of jet components. We calculate the minimal Lorentz factors for these sources from their measured apparent velocities, and their black hole masses are estimated with their broadline widths. A significant intrinsic correlation is found between black hole masses and the minimal Lorentz factors of the jet components, which the Eddington ratio is only weakly correlated with the minimal Lorentz factor, which may imply that the Blandford-Znajek (BZ) mechanism may dominate over the Blandford-Payne (BP) mechanism for the jet acceleration (at least) in blazars.
\end{abstract}

Key words: black hole physics — galaxies: active — galaxies: jets — galaxies: nuclei .

\section{INTRODUCTION}

Relativistic jets have been observed in many radio-loud AGNs, which are believed to be formed very close to the black holes. The currently most favored models of the jet

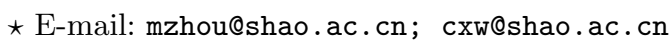


Ming Zhou and Xinwu Cao

formation are BZ and BP mechanisms (Blandford \& Znajek 1977; Blandford \& Payne 1982). In these mechanisms, the power of jet is extracted from the disk or black hole rotational energy. The disk-jet connection has been investigated by many authors in different ways (Rawlings \& Saunders 1991; Falcke \& Biermann 1995; Cao \& Jiang 1999; 2001; 2002, Xie et al. 2007; Xie et al. 2008).

Some different approaches were proposed to estimate the masses of the black holes in AGNs, such as the gas kinematics near a black hole (see Ho \& Kormendy 2000 for a review and references therein). The central black hole mass derived from the direct measurements on the gases moving near the hole is reliable, but unfortunately, it is available only for very few AGNs. For most AGNs, the velocities of the clouds in broad line regions (BLR) can be inferred from the widths of their broad emission lines. If the radius of the BLR is available, the mass of the central black hole can be derived from the broad-line width on the assumption that the clouds in the BLR are gravitationally bound and orbiting with Keplerian velocities (Dibai 1980). The radius of the BLR can be measured by using the reverberation-mapping method from the time delay between the continuum and line variations (Peterson 1993; Netzer \& Peterson 1997). Long-term monitoring on the source is necessary for applying this method to derive the radius of the BLR, which leads to a small amount of AGNs with measured black hole masses in this way. Alternatively, a tight correlation was found between the size of the BLR and the optical continuum luminosity, which can be used to estimate the size of the BLR in an AGN from its optical luminosity and then the black hole mass (e.g., Wandel, Peterson \& Malkan 1999; Kaspi et al. 1996; 2000; Laor 2000).

The kinematic properties of the jet components in blazars were revealed by multiepoch VLBI observations (e.g., Kellermann et al. 2004; Lister et al. 2005). In this paper, we use a large sample of blazars, of which the proper motions were well measured with VLBA, to explore the relations between the jet speeds and physical properties of blazars, i.e., the black hole masses and Eddington ratios.

The cosmological parameters $\Omega_{\mathrm{M}}=0.3, \Omega_{\Lambda}=0.7$, and $H_{0}=70 \mathrm{~km} \mathrm{~s}^{-1} \mathrm{Mpc}^{-1}$ have been adopted in this work.

\section{SAMPLE}

We start with a sample of radio-loud quasars and BL Lac objects with measured apparent velocities of jet components. The sample is compiled by searching the literature to include all blazars with available proper motion data of the jets. Most data are taken from several surveys, such as, the original flux-limited MOJAVE-I sample, and the extended

MOJAVE-II sample 1 . We find that 278 sources have multi-epoch VLBI observations, of which 146 blazars have reliable measured apparent velocities. Their black hole masses

${ }^{1}$ http://www.physics.purdue.edu/astro/MOJAVE 
are estimated with the broad-line widths and broad-line/continuum luminosities, which leads to 78 sources with measured black hole masses.

\section{BLACK HOLE MASSES AND MINIMAL LORENTZ FACTORS}

In order to estimate their $M_{\mathrm{BH}}$, we search the literatures for all the available measurements of the full width at half maximum (FWHM) for broad-lines $\mathrm{H} \alpha, \mathrm{H} \beta, \mathrm{Mg}$ II, C IV or Ly $\alpha$ lines, as well as the fluxes of these lines. For the sources without line flux data, we adopt their continuum fluxes instead. We find that one source in our sample has very narrow broad-lines $\left(\mathrm{FWHM}<1000 \mathrm{~km} \mathrm{~s}^{-1}\right)$, which is similar to typical narrow lines. It should be cautious on the black hole mass estimates for this source, since we cannot rule out the possibility that this line may be the narrow component emitted from the narrow line region (e.g., Gu et al. 2001). We therefore rule out this source. For most BL Lac objects, their broad-line emissions are too weak to be measured, and we estimate their $M_{\mathrm{BH}}$ with the empirical relation between $M_{\mathrm{BH}}$ and bulge luminosity $L_{\mathrm{bulge}}$. We list all the data of the sample in Table 1. Columns (1)-(2) represent the source's IAU name and redshift, respectively. The lines used to estimate $M_{\mathrm{BH}}$ from their luminosity and the references are listed in Columns (3) and (4), respectively. In Columns (5), we list the lines, of which the widths are used to estimate the $M_{\mathrm{BH}}$. We list the estimated $M_{\mathrm{BH}}$ in Columns (7). The data of the BL Lac objects in this sample are summarized in Table 2.

For blazars, the optical/UV continuum may be contaminated by the beamed synchrotron emission from the jets. Wu et al. (2004) compared the black hole masses obtained for a sample of radio-loud quasars with both the line and continuum, and they found that the masses obtained with line luminosity are systematically lower that those obtained with continuum. In this work, the black hole masses $M_{\mathrm{BH}}$ are estimated by using the line width of either one of these lines: $\mathrm{Mg}$ II, $\mathrm{H} \beta$, or $\mathrm{H} \alpha$, and the line luminosities (or the optical/UV continuum, if the line luminosity is unavailable). McGill et al. (2008) analyzed a sample of 19 AGNs of which all three lines were observed in optical wavebands, and they obtained a set of 30 internally self-consistent recipes for estimating $M_{\mathrm{BH}}$ from a variety of observables with different intrinsic scatters. Whenever more than one recipes are available for estimating the black hole mass, we always choose the one with the minimal intrinsic scatter (see McGill et al. 2008 for the details). We therefore use the broad-line emission instead of the optical/UV continuum to estimate $M_{\mathrm{BH}}$, provided their line luminosities are available. When more than one empirical correlations are applicable, we use one of following relations in order:

$$
\begin{aligned}
& \log M_{\mathrm{BH}}=6.384+2 \log \left(\frac{\mathrm{FWHM}_{\mathrm{Mg}_{\mathrm{II}}}}{1000 \mathrm{~km} \mathrm{~s}^{-1}}\right)+0.55 \log \left(\frac{L_{\mathrm{H} \alpha}}{10^{44} \mathrm{erg} \mathrm{s}^{-1}}\right) \\
& \log M_{\mathrm{BH}}=6.711+2 \log \left(\frac{\mathrm{FWHM}_{\mathrm{Mg}_{\mathrm{II}}}}{1000 \mathrm{~km} \mathrm{~s}^{-1}}\right)+0.56 \log \left(\frac{L_{\mathrm{H} \beta}}{10^{44} \mathrm{erg} \mathrm{s}^{-1}}\right)
\end{aligned}
$$




$$
\begin{aligned}
& \log M_{\mathrm{BH}}=6.711+2 \log \left(\frac{\mathrm{FWHM}_{\mathrm{Mg} \text { II }}}{1000 \mathrm{~km} \mathrm{~s}^{-1}}\right)+0.56 \log \left(\frac{L_{\mathrm{H} \alpha}^{\prime}}{10^{44} \mathrm{erg} \mathrm{s}^{-1}}\right), \\
& \log M_{\mathrm{BH}}=6.930+2 \log \left(\frac{\mathrm{FWHM}_{\mathrm{H} \alpha}}{1000 \mathrm{~km} \mathrm{~s}^{-1}}\right)+0.56 \log \left(\frac{L_{\mathrm{H} \beta}}{10^{44} \mathrm{erg} \mathrm{s}^{-1}}\right), \\
& \log M_{\mathrm{BH}}=6.747+2 \log \left(\frac{\mathrm{FWHM}_{\mathrm{H} \beta}}{1000 \mathrm{~km} \mathrm{~s}^{-1}}\right)+0.55 \log \left(\frac{L_{\mathrm{H} \beta}}{10^{44} \mathrm{erg} \mathrm{s}^{-1}}\right), \\
& \log M_{\mathrm{BH}}=6.420+2 \log \left(\frac{\mathrm{FWHM}_{\mathrm{H} \beta}}{1000 \mathrm{~km} \mathrm{~s}^{-1}}\right)+0.56 \log \left(\frac{L_{\mathrm{H} \alpha}}{10^{44} \mathrm{erg} \mathrm{s}^{-1}}\right), \\
& \log M_{\mathrm{BH}}=6.747+2 \log \left(\frac{\mathrm{FWHM}_{\mathrm{H} \beta}}{1000 \mathrm{~km} \mathrm{~s}^{-1}}\right)+0.55 \log \left(\frac{L_{\mathrm{H} \beta}^{\prime}}{10^{44} \mathrm{erg}_{, \mathrm{s}^{-1}}}\right) \\
& \log M_{\mathrm{BH}}=6.747+2 \log \left(\frac{\mathrm{FWHM}_{\mathrm{C}}{ }_{\mathrm{IV}}}{1000 \mathrm{~km} \mathrm{~s}^{-1}}\right)+0.55 \log \left(\frac{L_{\mathrm{H} \beta}^{\prime}}{10^{44} \mathrm{erg} \mathrm{s}^{-1}}\right)+\log 0.5 \\
& \log M_{\mathrm{BH}}=6.990+2 \log \left(\frac{\mathrm{FWHM}_{\mathrm{Mg}_{\mathrm{II}}}}{1000 \mathrm{~km} \mathrm{~s}^{-1}}\right)+0.518 \log \left(\frac{L_{5100}}{10^{42} \mathrm{erg} \mathrm{s}^{-1}}\right) \\
& \log M_{\mathrm{BH}}=7.026+2 \log \left(\frac{\mathrm{FWHM}_{\mathrm{H} \alpha}}{1000 \mathrm{~km} \mathrm{~s}^{-1}}\right)+0.518 \log \left(\frac{L_{5100}}{10^{42} \mathrm{erg} \mathrm{s}^{-1}}\right) \\
& \log M_{\mathrm{BH}}=7.026+2 \log \left(\frac{\mathrm{FWHM}_{\mathrm{C}}}{1000 \mathrm{~km} \mathrm{~s}^{-1}}\right)+0.518 \log \left(\frac{L_{5100}}{10^{42} \mathrm{erg} \mathrm{s}^{-1}}\right)+\log 0.5,
\end{aligned}
$$

where $M_{\mathrm{BH}}$ is in units of $M_{\odot}, L_{\mathrm{H} \alpha}^{\prime}$ and $L_{\mathrm{H} \beta}^{\prime}$ are estimate form $L_{\mathrm{Ly} \alpha}, L_{\mathrm{C}_{\mathrm{IV}}}$ or $L_{\mathrm{Mg} \text { II by }}$ their relative rations (Gaskell, Shields \& Wampler 1981; Francis et al. 1991), and $L_{5100}$ is nuclear luminosity $\lambda L_{\lambda}$ at $\lambda=5100 \AA$.

We also estimate $M_{\mathrm{BH}}$ using Eqs. (9), (10), and (11) for the black holes with continuum luminosities, of which the masses can also be estimated with line luminosities. We compare the black hole masses with these two different methods in Fig. 1. It is indeed found that the masses estimated with line luminosities are systematically lower that those estimated with continuum luminosities, which is consistent with Wu et al. (2004)'s conclusion.

For the BL Lac objects in this sample, we use the empirical relation between host galaxy absolute magnitude at R-band $M_{\mathrm{R}}$ and $M_{\mathrm{BH}}$ proposed by Bettoni et al. (2003),

$$
\log M_{\mathrm{BH}}=-0.50 M_{\mathrm{R}}-3.00
$$

to estimate their black hole masses.

Although the apparent velocities of the jet components were measured by VLBI observations, the intrinsic speeds of the jet components are still unavailable, as the viewing angles of the jets are unknown for most sources in this sample. However, we can derive the minimal Lorentz factors from the observed apparent velocities of jet components using:

$$
\gamma_{\min }=\left(1+\beta_{\mathrm{app}}^{2}\right)^{0.5}
$$

and then analyze their relations with other physical quantities of the sources. For the sources with more than one measured moving component, we always select the one moving fastest, as we intend to explore the acceleration mechanism of the jets in blazars (see Cohen et al. 2007 for the detailed discussion). 


\section{RESULTS}

In Fig. 2, we plot the relation between black hole masses $M_{\mathrm{BH}}$ and the minimal Lorentz factors $\gamma_{\min }$ of the jets. The linear regression gives

$$
\log \gamma_{\min }=0.31 \log M_{\mathrm{BH}}-1.80
$$

A significant correlation is found between these two quantities at 99.6 per cent confidence (Spearman rank correlation analysis), and the correlation coefficient is 0.33 . It should be noted with caution that this correlation may be caused by the common dependence of redshift. In Fig. 3, we plot the relation between redshift $z$ and the minimal Lorentz factor $\gamma_{\min }$, and only a weak correlation is found at 93.6 per cent confidence between these two quantities. We perform the Spearman partial rank correlation analysis (Macklin 1982), and we find that the partial correlation coefficient is 0.27 after subtracting the common redshift dependence. The significance of the partial rank correlation is 2.39, which is equivalent to the deviation from a unit variance normal distribution if there is no correlation present (see Macklin 1982 for the details). A summary of the results of partial rank correlation analysis is listed in Table 3.

We also perform a correlation analysis on the sources in the restricted redshift range $0.1<z<2.1$. For this subsample of 72 sources, a correlation at 97.6 per cent confidence is still present between $\gamma_{\min }$ and $M_{\mathrm{BH}}$, while almost no correlation between $\gamma_{\min }$ and $\mathrm{z}$ is found (at 55.2 per cent confidence). It appears that the correlation between $\gamma_{\min }$ and $M_{\mathrm{BH}}$ is an intrinsic one, not caused by the common redshift dependence.

The bolometric luminosity $\left(L_{\mathrm{bol}}\right)$ is estimated by assuming $L_{\mathrm{bol}} \approx 10 L_{\mathrm{BLR}}$ (e.g., Liu et al. 2006). For some sources without measured broad-line luminosities, we estimate the bolometric luminosities from the optical continuum luminosities using the relation of $L_{\mathrm{bol}} \approx 9 \lambda L_{\lambda \text {,opt }}(\lambda=5100 \AA)$ (Kaspi et al. 2000). We plot the relation between the Eddington ratio $\left(L_{\mathrm{bol}} / L_{\mathrm{Edd}}\right)$ and $\gamma_{\min }$ of the jets in Fig. 5. The linear regression gives

$$
\log \gamma_{\min }=0.11 \log L_{\mathrm{bol}} / L_{\mathrm{Edd}}+0.87
$$

We find that only a weak correlation between $L_{\mathrm{bol}} / L_{\mathrm{Edd}}$ and $\gamma_{\min }$ (at 93.5 per cent confidence) is present.

\section{DISCUSSION}

We find an intrinsic correlation between black hole masses and the minimal Lorentz factors of jet components for a sample of blazars, while no significant correlation between the Eddington rations and the Lorentz factors is present for the same sample. Our main statistical results will not be altered, even if those black holes with masses estimated with continuum luminosities are removed. Our statistical results provide useful clues to the mechanisms of jet formation and acceleration in blazars. 
It is believed that the growth of massive black holes in the centers of galaxies is dominantly governed by mass accretion in AGN phases (e.g., Soltan 1982; Yu \& Tremaine 2002). The massive black holes will be spun up through accretion, as the black holes acquire mass and angular momentum simultaneously though accretion. The spins of massive black holes may also be affected by the mergers of black holes. A rapidly rotating new black hole will be present after the merger of two black holes, only if the binary's larger member already spins quickly and the merger with the smaller hole is consistently near prograde, or if the binary's mass ratio approaches unity (Hughes \& Blandford 2003). The comoving space density for heavier black holes is much lower than that for lighter black holes (e.g., see the black hole mass function in Yu \& Tremaine 2002), which means that the probability of the mergers of two black holes with similar masses is lower for heavier black holes. This implies the spins of heavier black holes are mainly regulated by accretion rather than the mergers. Thus, it is natural to expect (in statistical sense) that the heavier black holes have higher spin parameters $a$ than their lower mass counterparts. Volonteri et al. (2007) studied on how the accretion from a warped disc influences the evolution of black hole spins and concluded that within the cosmological framework, one indeed expects most supermassive black holes in elliptical galaxies to have on average higher spin than black holes in spiral galaxies, where random, small accretion episodes (e.g., tidally disrupted stars, accretion of molecular clouds) might have played a more important role. The jets can be accelerated to higher speeds by the heavier black holes, because they are spinning more rapidly (Blandford \& Znajek 1977). The intrinsic correlation between black hole masses and the minimal Lorentz factors of jet components found in this work is consistent with the Blandford-Znajek mechanism. The properties of accretion disks accretion disk are related with the dimensionless accretion rates $\dot{m}$ $\left(\dot{m}=\dot{M} / \dot{M}_{\mathrm{Edd}} \propto L_{\mathrm{bol}} / L_{\mathrm{Edd}}\right)$. No significant correlation between $L_{\mathrm{bol}} / L_{\mathrm{Edd}}$ and $\gamma_{\text {min }}$ is found, which implies that the jet acceleration may not be related with the properties of the accretion disk, which may imply that the jet formation is not sensitive to the disk structure. This is, of course, quite puzzling, and to be verified by the future work with a larger blazar sample. Our statistical results implies that the BZ mechanism may dominate over BP mechanism for the jet acceleration in blazars.

Acknowledgements We thank the referee for the helpful comments/suggestions, and 'MOJAVE survey' for sharing their data on the website. This work is supported by the NSFC (10773020), and the CAS (grant KJCX2-YW-T03). This research has made use of the NASA/IPAC Extragalactic Database (NED), which is operated by the Jet Propulsion Laboratory, California Institute of Technology, under contract with the National Aeronautics and Space Administration. 


\section{References}

Baldwin J.A., Wampler E.J., Gaskell C.M., 1989, ApJ, 338, 630 (B89)

Bettoni, D., Falomo, R., Fasano,G., et al., 2003, A\&A, 339, 869

Blandford R. D., Payne D. G., 1982, MNRAS, 199, 883

Blandford R. D., Znajek R. L., 1977, MNRAS, 179, 433

Brotherton M. S. 1996, ApJS, 102, 1 (B96)

Cao X., Jiang D. R., 1999, MNRAS, 307, 802

Cao X., Jiang D. R., 2001, MNRAS, 320, 347

Cao X., Jiang D. R., 2002, MNRAS, 331, 111

Cohen M. H., Lister M. L., Homan D. C., et al., 2007, ApJ, 658, 232

Dibai, É. A. 1980, Azh., 57, 677 (English translation: Sov. Astron., 24, 389)

Falcke H., Biermann P., 1995, A\&A, 293, 665

Francis P. J., Hewett P. C., Foltz C. B., et al., 1991, ApJ, 373, 465

Gaskell C. M., Wampler E. J., Shields G. A., 1981, ApJ, 249, 443

Gelderman R., Whittle M. 1994, ApJS, 91, 491 (G94)

Gu M., Cao X., Jiang D. R., 2001, MNRAS, 327, 1111

Henstock D. R., Browne I. W. A., Wilkinson P. N., et al., 1997,MNRAS, 290, 380 (H97)

Ho L. C., Kormendy J., 2000, The Encyclopedia of Astronomy and Astrophysics (Institute of Physics Publishing). (astro-ph/0003267)

Hughes S. A., Blandford Roger D. 2003, ApJ, 585, L101

Hunstead R. W., Murdoch H. S., Shobbrook R. R., 1978, MNRAS, 185, 149(H78)

Jackson N., Browne W. A. 1991, MNRAS, 250, 414 (JB91a)

Jackson N., Browne W. A. 1991, MNRAS, 250, 422 (JB91b)

Kaspi S., Smith P. S., Maoz D., et al., 1996, ApJ, 471, L75

Kaspi S., Smith P. S., Netzer H., et al., 2000, ApJ, 533, 631

Kellermann, K. I., Lister, M. L., D. C. Homan, 2004, ApJ, 609, 539

Laor A. 2000, ApJ, 543, L111

Lawrence C. R., Zucker J. R., REanhead C. S. et al. 1996, ApJS, 107, 541 (L96)

Lister M. L., Homan D. C., 2005, AJ, 130, 1389L

Liu Y., Jiang D. R.,Gu M. F., 2006, ApJ, 637, 669

Macklin J. T., 1982, MNRAS, 199, 1119

Marziani P., Sulentic J. W., Dultzin-Hacyan D. et al. 1996, ApJS, 104, 37 (M96)

McGill K. L., Woo J.-H., Treu T., et al., 2008, ApJ, 673,703

Nerzer H. N., Brotherton M. S., Wills B. J. et al. 1995, ApJ, 448, 27 (N95)

Netzer H., Peterson B.M., 1997, in Astronomical Time Series, eds., D. Maoz, A. Sternberg \& E. Leibowitz (Dordrecht: Kluwer), 85

Neugebauer G., Oke J. B., Beckline E. E., Matthews K.,1979, ApJ, 230, 79 (N79)

Oshlack A. Y. K. N., Webster R. L., Whiting M. T. 2002, ApJ, 576, 81 (O02)

Peterson B. M., 1993, PASP, 105, 207

Rawlings S. G., Saunders R. D. E., 1991, Nat, 349, 138

Scarpa R., Falomo R., 1997, A\&A, 325, 109 (S97)

Soltan A. 1982, MNRAS, 200, 115

Steidel C. C., Sargent W. L. W. 1991, ApJ, 382, 433 (S91)

Stickel M., Fried W., Kühr H., 1989, A\&AS, 80, 103 (S89)

Stickel M., Kühr H., 1993, A\&AS, 100, 395 (SK93)

Stickel M., Kühr H., Fried J. W. 1993, A \& AS, 97, 483 (S93)

Tadhunter C. N., Morganti R., Alighieri S. S. et al. 1993, MNRAS, 263, 999 (T93)

Urry C. M., Scarpa, R., O'Dowd M., et al., 2000, ApJ, 532, 816 (U00)

Volonteri M., Sikora M., Lasota J.-P., 2007, ApJ, 667, 704

Wandel A., Peterson B.M., Malkan M.A., 1999, ApJ, 526, 579

Wills B., Browne I. W. A. 1986, ApJ, 302, 56 (W86)

Wills B. J., Thompson K. L., Han M., et al., 1995, ApJ, 447, 139 (W95) 
Wu X. B., Wang R., Kong M. Z., et al., 2004, A\&A, 424, 793

Xie G. Z., Dai H., Zhou S. B., 2007, AJ, 134, 1464

Xie Z. H., Hao J. M., Du L. M., Zet al., 2008, PASP, 120, 477

Yu Q., Tremaine S. 2002, MNRAS, 335, 965 
Table 1 The data for quasars. Col. (1): IAU source name. Col. (2): Redshift.

Col. (3): $\log$ of the minimal Lorentz factor of jet. Col. (4): the lines used to estimate the black hole masses from their fluxes. Col. (5): references for Col. (4). Col. (6): the lines used to estimate the black hole masses from their FWHMs. Col. (7): references for Col. (6). Col. (8): the black hole masses.

\begin{tabular}{|c|c|c|c|c|c|c|c|}
\hline $\begin{array}{l}\text { Source } \\
\text { (1) }\end{array}$ & $\begin{array}{l}\mathrm{z} \\
(2)\end{array}$ & $\begin{array}{c}\log \gamma_{\min } \\
(3)\end{array}$ & $\begin{array}{l}\text { line } \\
(4)\end{array}$ & $\begin{array}{l}\text { Refs. } \\
(5)\end{array}$ & $\begin{array}{l}\text { line } \\
(6)\end{array}$ & $\begin{array}{l}\text { Refs. } \\
(7)\end{array}$ & $\begin{array}{c}\log M_{\mathrm{BH}} / M_{\odot} \\
(8)\end{array}$ \\
\hline $0016+731$ & 1.781 & 0.760 & $\mathrm{Mg}$ II & L96 & Ly $\alpha \mathrm{C}$ IV Mg II & L96 & 8.93 \\
\hline $0035+413$ & 1.353 & 0.926 & Mg II & SK93 & Mg II & SK93 & 8.53 \\
\hline $0106+013$ & 2.107 & 1.461 & C IV & B89 & C IV & B89 & 8.83 \\
\hline $0112-017$ & 1.365 & 0.159 & $\mathrm{Mg}$ II & B89 & C IV & B89 & 7.85 \\
\hline $0119+041$ & 0.637 & 0.291 & $\mathrm{H} \beta$ & JB91b & $\mathrm{H} \beta$ & JB91a & 8.50 \\
\hline $0133+476$ & 0.859 & 0.350 & $\mathrm{Mg}$ II & L96 & $\mathrm{H} \beta$ & L96 & 8.30 \\
\hline $0212+735$ & 2.367 & 1.071 & $\mathrm{Mg}$ II & L96 & Lya C IV Mg II & L96 & 8.48 \\
\hline $0333+321$ & 1.263 & 1.030 & Mg II & B94 & Mg II & S91 & 8.49 \\
\hline 0336-019 & 0.852 & 1.006 & $\mathrm{Mg}$ II & B89 & $\mathrm{H} \beta$ & JB91a & 8.78 \\
\hline $0403-132$ & 0.571 & 1.279 & $\mathrm{H} \beta$ & M96 & $\mathrm{H} \beta$ & S97 & 8.77 \\
\hline $0420-014$ & 0.915 & 0.933 & $\mathrm{Mg}$ II & B89 & Mg II & S97 & 8.84 \\
\hline $0440-003$ & 0.844 & 0.183 & $\mathrm{Mg}$ II & B89 & $\mathrm{H} \beta$ & JB91a & 8.63 \\
\hline $0605-085$ & 0.872 & 1.367 & $\mathrm{Mg}$ II & S93 & Mg II & S93 & 8.43 \\
\hline $0607-157$ & 0.324 & 0.047 & $\mathrm{H} \beta$ & $\mathrm{H} 78$ & $\mathrm{H} \beta$ & $\mathrm{H} 78$ & 7.63 \\
\hline $0736+017$ & 0.191 & 1.082 & $\mathrm{H} \beta$ & B96 & $\mathrm{H} \beta$ & S97 & 8.23 \\
\hline $0738+313$ & 0.630 & 0.892 & $\mathrm{H} \beta$ & B96 & $\mathrm{H} \beta$ & JB91a & 9.08 \\
\hline $0804+499$ & 1.432 & 0.552 & $\mathrm{Mg}$ II & L96 & C IV Mg II & L96 & 8.57 \\
\hline $0836+710$ & 2.180 & 1.550 & $\mathrm{Mg}$ II & L96 & Ly $\alpha \mathrm{C}$ IV Mg II & L96 & 9.49 \\
\hline $0850+581$ & 1.322 & 0.903 & $\mathrm{Mg}$ II & L96 & $\mathrm{Mg}$ II & L96 & 9.67 \\
\hline $0859-140$ & 1.339 & 1.214 & $\mathrm{Mg}$ II & B94 & Mg II & S91 & 8.87 \\
\hline $0906+015$ & 1.018 & 1.288 & $\mathrm{Mg}$ II & B89 & $\mathrm{Mg}$ II & S97 & 8.63 \\
\hline $0923+392$ & 0.698 & 0.729 & $\mathrm{Mg}$ II & L96 & $\mathrm{H} \beta$ & L96 & 9.27 \\
\hline $0945+408$ & 1.252 & 1.230 & $\mathrm{Mg}$ II & L96 & C IV Mg II & L96 & 9.71 \\
\hline $0953+254$ & 0.712 & 1.063 & $\mathrm{H} \beta$ & JB91b & $\mathrm{H} \beta$ & JB91a & 8.73 \\
\hline $1038+064$ & 1.265 & 0.848 & $\mathrm{Mg}$ II & B94 & Mg II & S91 & 8.76 \\
\hline $1055+018$ & 0.888 & 0.398 & Mg II & B89 & Mg II & S97 & 8.45 \\
\hline $1226+023$ & 0.158 & 1.118 & $\mathrm{Mg}$ II & B89 & $\mathrm{H} \alpha$ & JB91a & 8.76 \\
\hline $1253-055$ & 0.538 & 0.953 & $\mathrm{Mg}$ II & W95 & $\mathrm{H} \alpha$ & N79 & 8.53 \\
\hline $1302-102$ & 0.278 & 0.744 & $\mathrm{Mg}$ II & B89 & $\mathrm{H} \beta$ & M96 & 7.90 \\
\hline $1334-127$ & 0.539 & 1.247 & $\mathrm{Mg}$ II & S93 & $\mathrm{Mg}$ II & S93 & 8.36 \\
\hline $1458+718$ & 0.904 & 0.833 & Mg II & L96 & $\mathrm{H} \beta$ & L96 & 8.84 \\
\hline $1502+106$ & 1.839 & 1.249 & Mg II & W86 & $\mathrm{C}$ IV & S97 & 8.86 \\
\hline $1504-166$ & 0.876 & 0.608 & Mg II & $\mathrm{H} 78$ & Mg II & $\mathrm{H} 78$ & 8.84 \\
\hline $1510-089$ & 0.360 & 1.133 & $\mathrm{Mg}$ II & W86 & $\mathrm{H} \alpha$ & N79 & 8.22 \\
\hline $1532+016$ & 1.420 & 1.147 & Mg II & B89 & C IV Mg II & S97 & 8.73 \\
\hline
\end{tabular}


Table 1 Continued...

\begin{tabular}{|c|c|c|c|c|c|c|c|}
\hline $\begin{array}{l}\text { Source } \\
\text { (1) }\end{array}$ & $\begin{array}{c}\mathrm{Z} \\
(2)\end{array}$ & $\begin{array}{c}\log \gamma_{\min } \\
(3)\end{array}$ & $\begin{array}{l}\text { line } \\
(4)\end{array}$ & $\begin{array}{c}\text { Refs. } \\
(5)\end{array}$ & $\begin{array}{l}\text { line } \\
(6)\end{array}$ & $\begin{array}{l}\text { Refs. } \\
(7)\end{array}$ & $\begin{array}{c}\log M_{\mathrm{BH}} / M_{\odot} \\
(8)\end{array}$ \\
\hline $1546+027$ & 0.412 & 1.071 & $\mathrm{Mg}$ II & B89 & $\mathrm{H} \beta$ & $\mathrm{S} 97$ & 8.82 \\
\hline $1611+343$ & 1.401 & 1.197 & $\mathrm{H} \beta$ & N95 & Ly $\alpha \mathrm{C}$ IV & W95 & 9.49 \\
\hline $1633+382$ & 1.807 & 1.380 & Mg II & L96 & Ly $\alpha \mathrm{C}$ IV $\mathrm{Mg}$ II & L96 & 10.14 \\
\hline $1637+574$ & 0.751 & 1.118 & $\mathrm{Mg}$ II & L96 & $\mathrm{H} \beta$ & L96 & 8.68 \\
\hline $1641+399$ & 0.594 & 1.275 & $\mathrm{Mg}$ II & L96 & $\mathrm{H} \beta$ & L96 & 9.03 \\
\hline $1642+690$ & 0.751 & 1.222 & Mg II & L96 & $\mathrm{Mg}$ II & L96 & 8.49 \\
\hline $1656+053$ & 0.879 & 0.655 & $\mathrm{H} \beta$ & B96 & $\mathrm{Mg}$ II & $\mathrm{S} 97$ & 9.09 \\
\hline $1739+522$ & 1.379 & 0.961 & $\mathrm{C}$ IV & L96 & C IV & L96 & 8.20 \\
\hline $1741-038$ & 1.057 & 0.827 & $\mathrm{Mg}$ II & S89 & $\mathrm{Mg}$ II & S89 & 8.67 \\
\hline $1828+487$ & 0.692 & 1.110 & $\mathrm{Mg}$ II & L96 & $\mathrm{H} \beta$ & L96 & 8.66 \\
\hline $1921-293$ & 0.352 & 0.637 & $\mathrm{H} \beta$ & JB91b & $\mathrm{H} \alpha$ & JB91a & 8.38 \\
\hline $1928+738$ & 0.303 & 0.913 & $\mathrm{H} \alpha$ & L96 & $\mathrm{H} \beta$ & L96 & 8.76 \\
\hline $2113+293$ & 1.514 & 0.303 & $\mathrm{Mg}$ II & $\mathrm{S} 93$ & $\mathrm{Mg}$ II & $\mathrm{S} 93$ & 8.74 \\
\hline $2121+053$ & 1.941 & 1.165 & $\mathrm{Mg}$ II & B94 & $\mathrm{Mg}$ II & $\mathrm{S} 91$ & 8.60 \\
\hline $2128-123$ & 0.501 & 0.864 & $\mathrm{H} \alpha$ & $\mathrm{O} 02$ & $\mathrm{H} \beta$ & T93 & 9.16 \\
\hline $2134+004$ & 1.932 & 0.391 & $\mathrm{C}$ IV & B89 & $\mathrm{C}$ IV & B89 & 8.50 \\
\hline $2145+067$ & 0.999 & 0.407 & $\mathrm{Mg}$ II & B94 & $\mathrm{Mg}$ II & $\mathrm{S} 91$ & 8.61 \\
\hline $2155-152$ & 0.672 & 0.523 & Mg II & S89 & $\mathrm{H} \beta$ & $\mathrm{S} 89$ & 7.81 \\
\hline $2201+315$ & 0.298 & 0.848 & Mg II & W95 & $\mathrm{H} \alpha$ & JB91a & 8.91 \\
\hline $2216-038$ & 0.901 & 0.748 & Mg II & B94 & Ly $\alpha \mathrm{C}$ IV Mg II & W95 S91 & 8.89 \\
\hline $2223-052$ & 1.404 & 1.249 & $\mathrm{C}$ IV & W95 & Ly $\alpha \mathrm{C}$ IV $\mathrm{Mg}$ II & W95 S97 & 8.54 \\
\hline $2230+114$ & 1.037 & 0.950 & C IV & W95 & Ly $\alpha \mathrm{C}$ IV Mg II & W95 S97 & 8.64 \\
\hline $2251+158$ & 0.859 & 1.187 & $\mathrm{H} \beta$ & N95 & $\mathrm{H} \beta$ & JB91a & 8.87 \\
\hline $2345-167$ & 0.576 & 1.145 & $\mathrm{H} \beta$ & JB91b & $\mathrm{H} \beta$ & JB91a & 8.59 \\
\hline $2351+456$ & 1.986 & 1.452 & $\mathrm{Mg}$ II & L96 & $\mathrm{Mg}$ II & L96 & 9.22 \\
\hline $0458-020$ & 2.291 & 1.179 & $\mathrm{C}$ IV & B89 & $m_{\mathrm{B}}$ & $\cdots$ & 9.27 \\
\hline $0730+504$ & 0.720 & 1.236 & $\mathrm{Mg}$ II & $\mathrm{H} 97$ & $m_{\mathrm{B}}$ & $\ldots$ & 8.84 \\
\hline $0748+126$ & 0.889 & 1.317 & $\mathrm{Mg}$ II & W86 & $m_{\mathrm{B}}$ & $\cdots$ & 8.84 \\
\hline $1012+232$ & 0.565 & 1.012 & $\mathrm{H} \beta$ & B96 & $m_{\mathrm{B}}$ & $\cdots$ & 8.69 \\
\hline $1127-145$ & 1.187 & 1.133 & Mg II & W86 & $m_{\mathrm{B}}$ & $\ldots$ & 9.18 \\
\hline $1145-071$ & 1.342 & 0.433 & C IV & W86 & $m_{\mathrm{B}}$ & $\ldots$ & 8.61 \\
\hline $1156+295$ & 0.729 & 1.290 & $\mathrm{H} \beta$ & B96 & $m_{\mathrm{B}}$ & $\ldots$ & 9.19 \\
\hline $1508-055$ & 1.191 & 1.269 & $\mathrm{Mg}$ II & W86 & $m_{\mathrm{B}}$ & $\ldots$ & 8.97 \\
\hline $1655+077$ & 0.621 & 1.048 & Mg II & W86 & $m_{\mathrm{B}}$ & $\cdots$ & 7.91 \\
\hline $1726+455$ & 0.714 & 0.602 & Mg II & $\mathrm{H} 97$ & $m_{\mathrm{B}}$ & $\cdots$ & 8.59 \\
\hline $1901+319$ & 0.635 & 0.455 & $\mathrm{H} \beta$ & G94 & $m_{\mathrm{B}}$ & $\cdots$ & 8.80 \\
\hline $2008-159$ & 1.178 & 0.536 & Mg II & W86 & $m_{\mathrm{B}}$ & $\cdots$ & 9.56 \\
\hline $2227-088$ & 1.562 & 0.925 & C IV & W86 & $m_{\mathrm{B}}$ & $\ldots$ & 8.85 \\
\hline
\end{tabular}

*the sources without line luminosities, we use their optical continuum luminosities at B band to 
References:B89: Baldwin et al. (1989). B94: Brotherton et al. (1996). B96: Brotherton (1996). G94: Gelderman et al. (1994). H78: Hunstead et al. (1978). H97: Henstock et al. (1997) JB91a: Jackson \&Browne (1991). JB91b: Jackson \& Browne (1991). L96: Lawrence et al. M96: Marziani et al. (1996). N79: Neugebauer et al. (1979). N95: Nerzer et al. (1995). O02: Oshlack et al. (2002). S89: Stickel et al. (1989). S91: Steidel et al. (1991). SK93: Stickel \& Kühr (1993). S93: Stickel et al. (1993). S97: Scarpa et al. (1997) T93: Tadhunter et al. (1993). W95: Wills et al. (1995). W86: Wills et al. (1986). 
Table $2 \gamma_{\min }$ and $M_{\mathrm{BH}}$ for BL Lac. Col. (1): IAU source name. Col. (2): Redshift. Col. (3): $\log$ of the minimal Lorentz factor of jet. Col. (4): absolute R host galaxy magnitude and the references, respectively. Col. (6): the black hole masses.

\begin{tabular}{|c|c|c|c|c|c|}
\hline $\begin{array}{l}\text { Source } \\
\text { (1) }\end{array}$ & $\begin{array}{c}\mathrm{z} \\
(2)\end{array}$ & $\begin{array}{c}\log \gamma_{\min } \\
(3)\end{array}$ & $\begin{array}{c}M_{\mathrm{R}}(\text { host }) \\
(4)\end{array}$ & $\begin{array}{c}\text { Refs. } \\
(5)\end{array}$ & $\begin{array}{c}\log M_{\mathrm{BH}} / M_{\odot} \\
(6)\end{array}$ \\
\hline $0829+046$ & 0.180 & 1.037 & -22.98 & U00 & 8.49 \\
\hline $1749+096$ & 0.320 & 0.923 & -22.68 & U00 & 8.34 \\
\hline $1807+698$ & 0.051 & 0.480 & -23.18 & U00 & 8.59 \\
\hline $2007+777$ & 0.342 & 0.114 & -22.96 & U00 & 8.48 \\
\hline $2200+420$ & 0.069 & 0.813 & -22.84 & U00 & 8.42 \\
\hline
\end{tabular}

References:U00: Urry et al. (2000). 
Table 3 The Spearman partial rank correlation analysis of the sample. Here $r_{\mathrm{AB}}$ is the rank correlation coefficient of the two variables, and $r_{\mathrm{AB}, \mathrm{C}}$ the partial rank correlation coefficient. The significance of the partial rank correlation is equivalent to the deviation from a unit variance normal distribution if there is no correlation present.

\begin{tabular}{|c|c|c|c|c|c|c|}
\hline Sample & $\mathrm{N}$ & Correlated variables: A,B & Variable: $\mathrm{C}$ & $r_{\mathrm{AB}}$ & $r_{\mathrm{AB}, \mathrm{C}}$ & significance \\
\hline \multirow[t]{4}{*}{ All } & 78 & $M_{\mathrm{BH}}, \gamma_{\min }$ & $\mathrm{z}$ & 0.33 & 0.27 & 2.39 \\
\hline & & $\mathrm{z}, \gamma_{\min }$ & $M_{\mathrm{BH}}$ & 0.21 & 0.07 & 0.61 \\
\hline & & $M_{\mathrm{BH}}, \mathrm{z}$ & $\gamma_{\min }$ & 0.46 & 0.42 & 3.85 \\
\hline & & $L_{\mathrm{bol}} / L_{\mathrm{Edd}}, \gamma_{\min }$ & $\mathrm{z}$ & 0.21 & 0.18 & 1.52 \\
\hline Within & 72 & $M_{\mathrm{BH}}, \gamma_{\min }$ & $\mathrm{z}$ & 0.27 & 0.25 & 2.14 \\
\hline \multirow[t]{2}{*}{$0.1<z<2.1$} & & $\mathrm{z}, \gamma_{\min }$ & $M_{\mathrm{BH}}$ & 0.09 & -0.02 & -0.17 \\
\hline & & $M_{\mathrm{BH}}, \mathrm{z}$ & $\gamma_{\min }$ & 0.41 & 0.40 & 3.47 \\
\hline
\end{tabular}




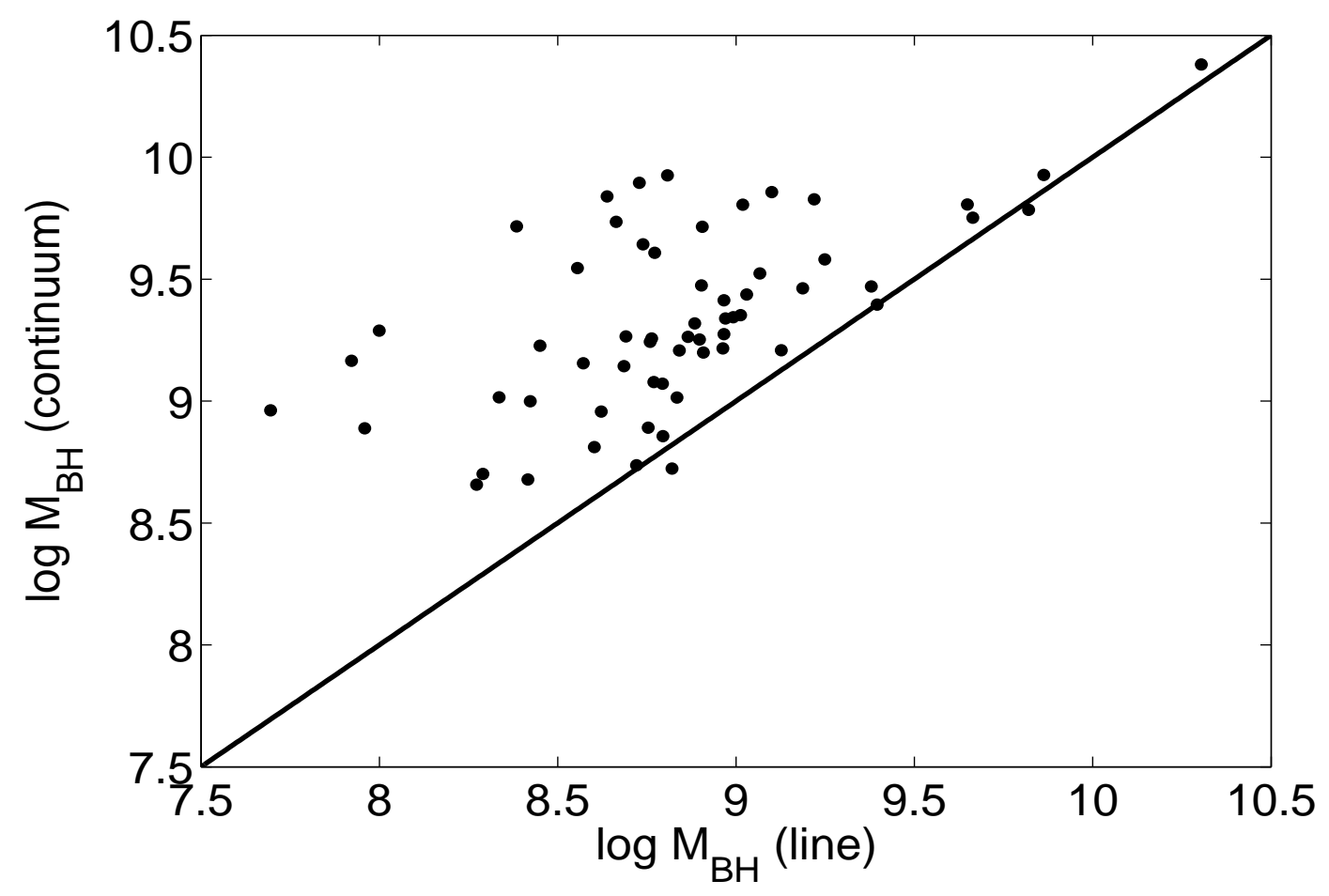

Fig. 1 The comparison of the black hole masses estimated with two different approaches. 


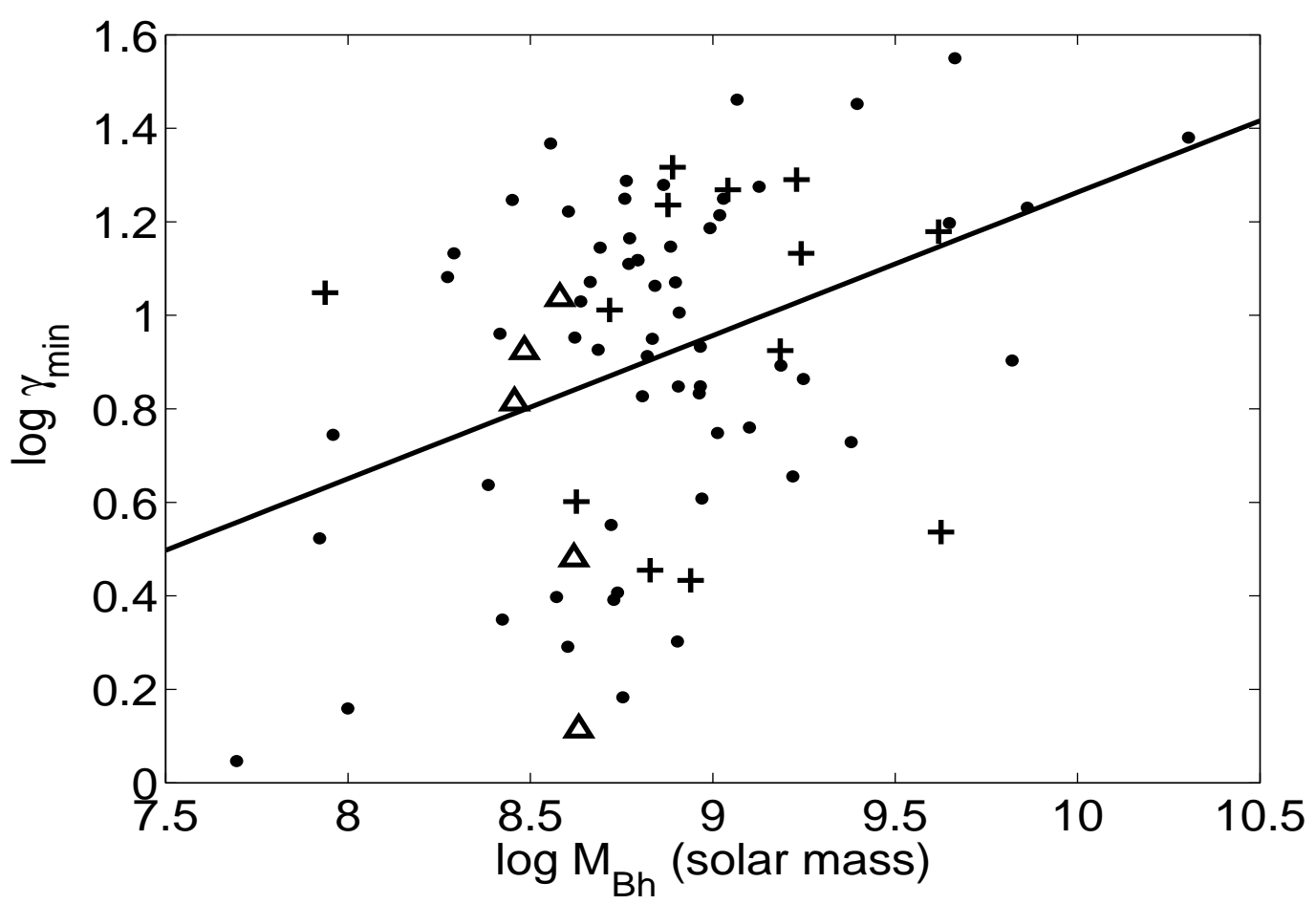

Fig. 2 The relation between black hole mass and the minimal Lorentz factor of the jet. The full circles represent quasars which $M_{\mathrm{BH}}$ estimated by line luminosities, while the triangles represent BL Lac objects. The crosses represent quasars which $M_{\mathrm{BH}}$ estimated by continuum luminosities. 


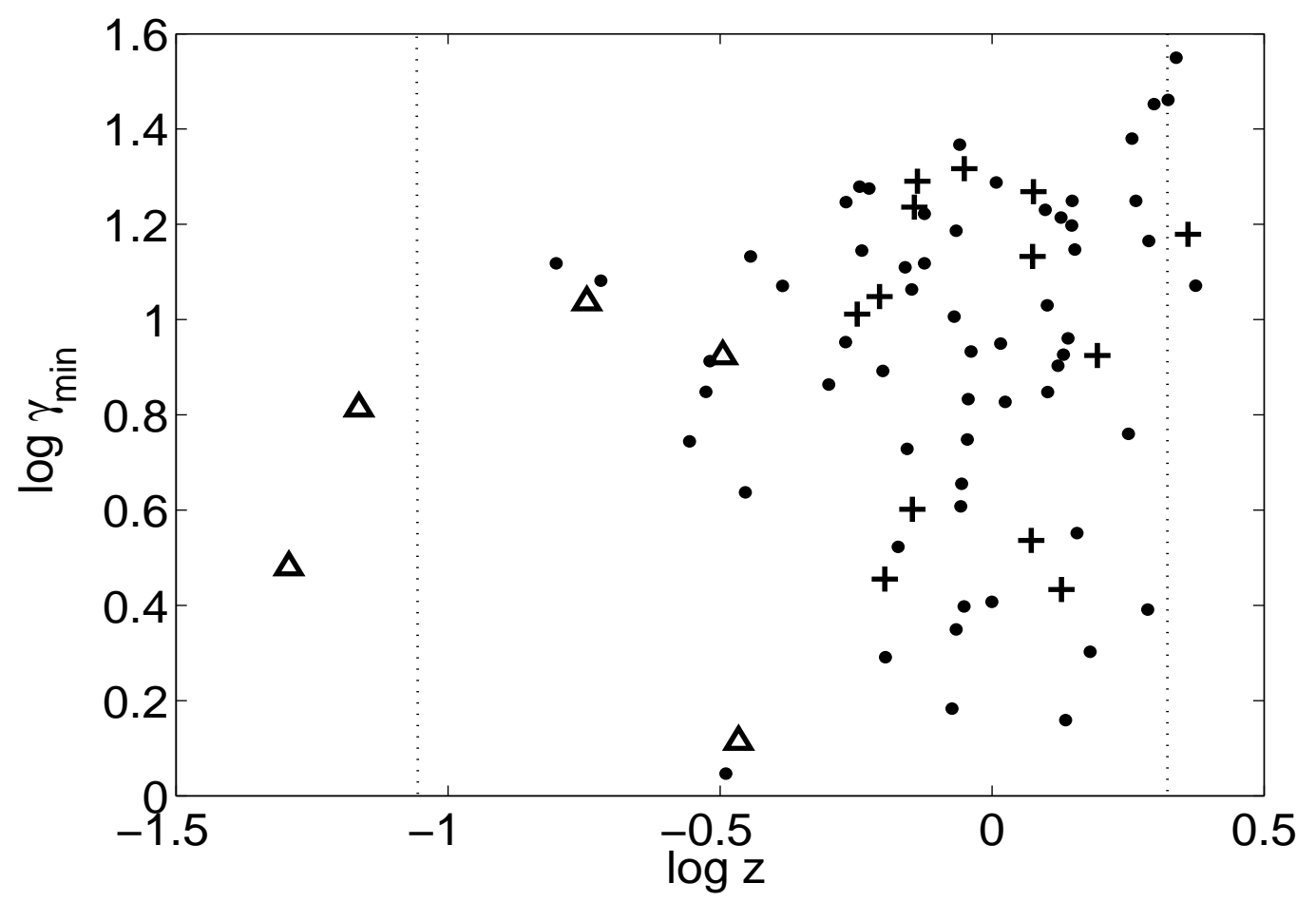

Fig. 3 The minimal Lorentz factor of jet versus redshift plane for our sample (symbols as in Fig. 2). The restricted redshift range, $0.1<z<2.1$, is indicated by the dotted lines. 


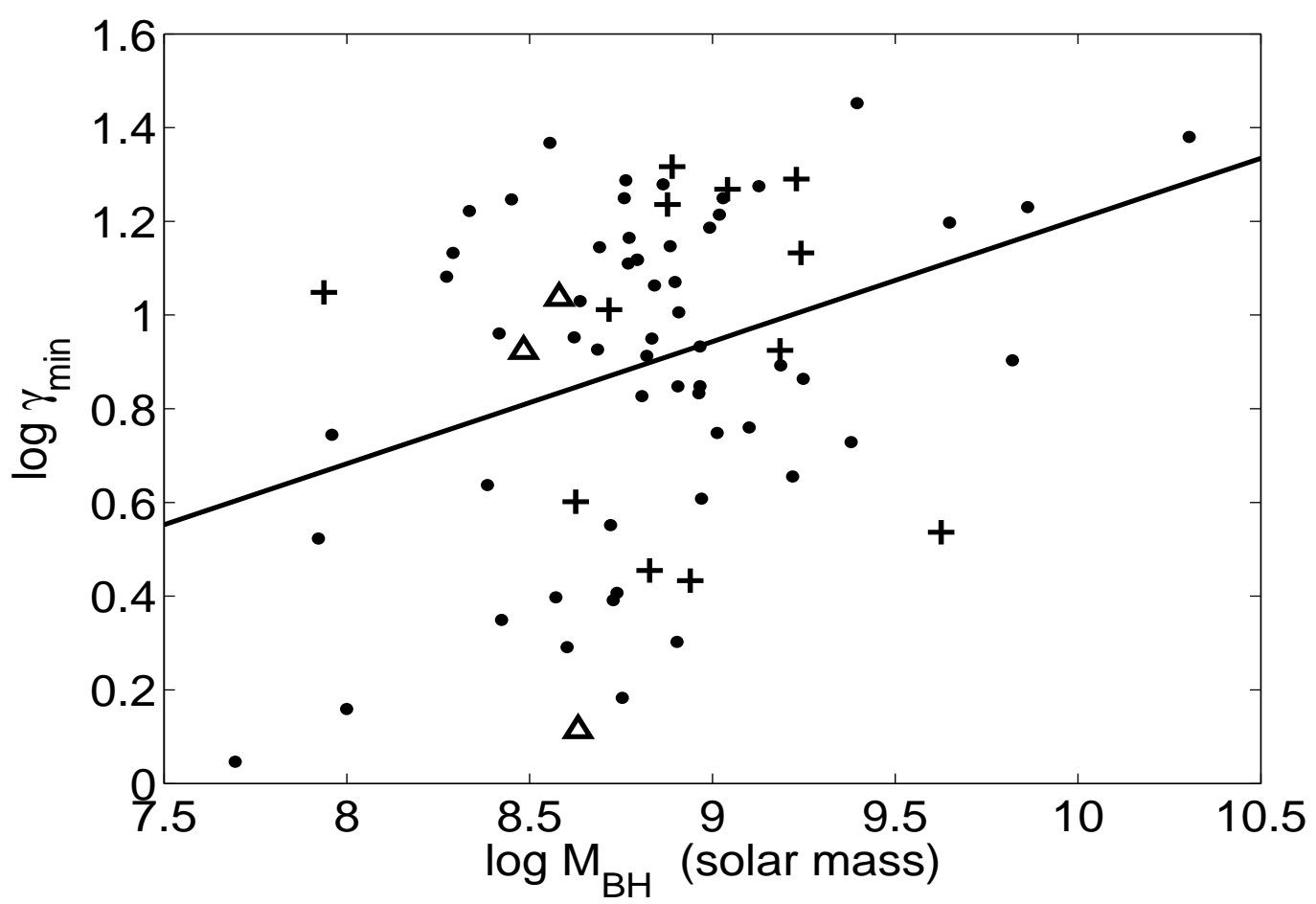

Fig. 4 The same as Fig. 2, but for the subsample within the restricted redshift range. 


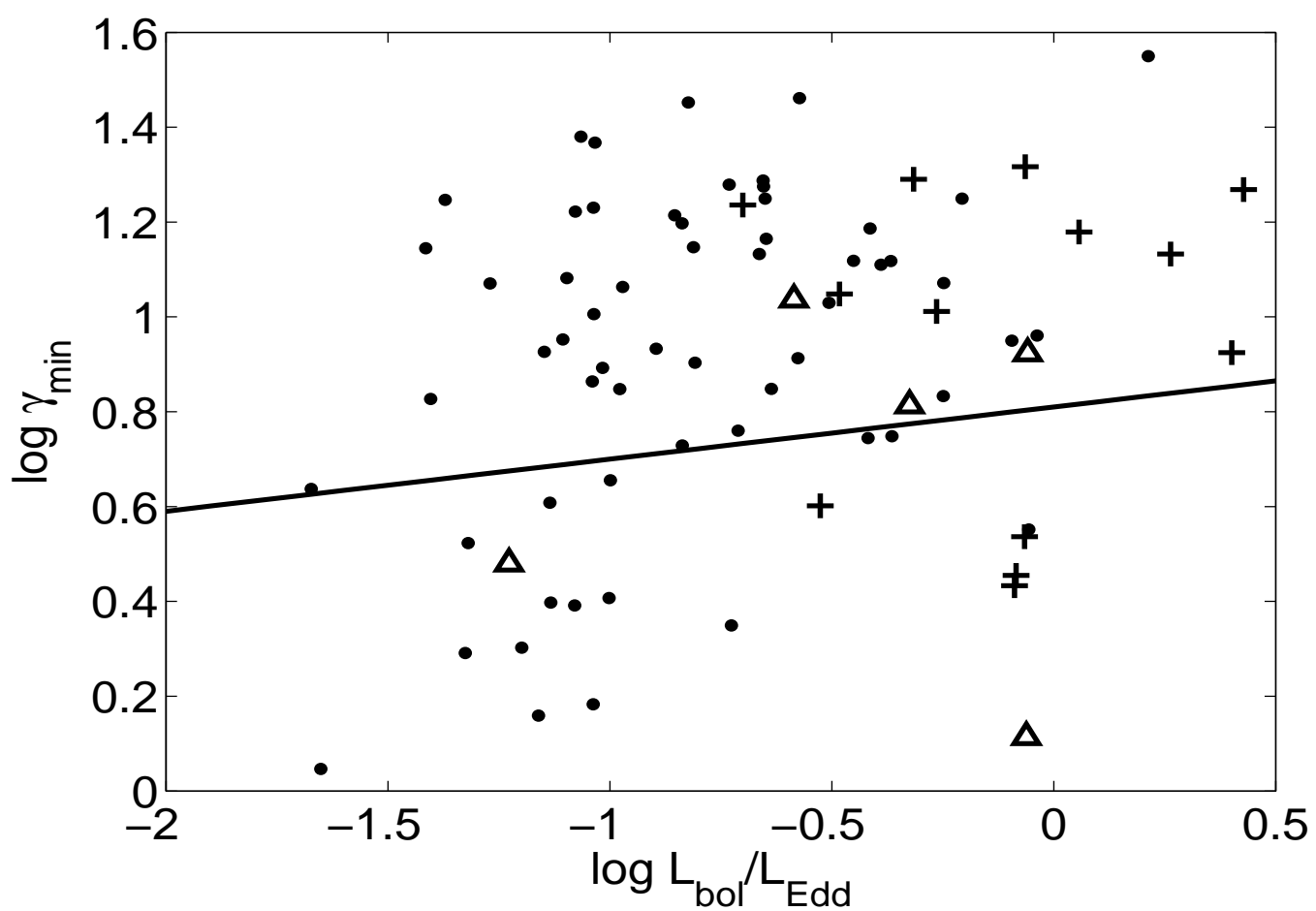

Fig. 5 The relation between the Eddington ratio and the minimal Lorentz factor of the jet (symbols as in Fig. 2). 